\title{
La aplicación de métodos tecnológicos de evaluación de idioma extranjero en relación con el nivel de estrés que estos generan en los estudiantes universitarios.
}

\section{The assessment of foreign language through technological applications and the stress level that they generate in college students.}

Edison Gerardo Llerena Medina. ${ }^{1}$, Carlos Patricio Rodríguez Hurtado. ${ }^{2}$ \& Edison Roberto Valencia Núñez. ${ }^{3}$

\begin{abstract}
The purpose of this research has been to find out the level of academic stress and its different behaviors, which occur when applying assessments to students using different technological tools such as StarQuiz Netclient, Socrative, Google Forms as well as tests through the Moodle platform of the Universidad Técnica de Ambato. Each of the tests evaluates reading comprehension skills as well as vocabulary knowledge in general and contains answer keys to be selected through mobile devices such as tablets, cell phones or personal computers. These tests are accompanied by three self-assessment tests to find out the level of stress, the first one before the test, the second one during the test and the third one after the test. This research was conducted at the Language Center of the Universidad Tecnica de Ambato, where it was applied to a sample of 215 students, who were studying English, Italian, French and Mandarin Chinese. For the data collection, the survey techniques applied in the test were used, in which the information generated was tabulated and represented by graphs and statistical tables; These results showed us a significant negative
\end{abstract}

\footnotetext{
${ }^{1}$ Universidad Técnica de Ambato, Facultad de Ciencia de la Educación, Ambato, Ecuador, eg.llerena@uta.edu.ec

2 Universidad Técnica de Ambato, Facultad de Ciencia de la Educación, Ambato, Ecuador, cp.rodriguez@uta.edu.ec

${ }^{3}$ Universidad Técnica de Ambato, Facultad de Ciencia de la Educación, Ambato, Ecuador, edisonvalencia@uta.edu.ec
} 
correlation between the level of stress generated and academic performance, and that there is a greater degree of stress during the test, lowering the level of stress before the test and a minimum level of stress at the end of the test; which implies that students face an evaluation using technology there is little tolerance of behaviors against the evaluations they perform.

Key Words: Technological apps, Academic Stress, academic performance, online assessment.

\section{RESUMEN}

La presente investigación tuvo por objeto averiguar el nivel de estrés académico y sus diferentes comportamientos, que se producen al aplicar evaluaciones a los estudiantes usando algunas herramientas tecnológicas como son StarQuiz Netclient, Socrative, Google Forms como también pruebas plateadas a través de la plataforma Moodle de la Universidad Técnica de Ambato. Cada una de las pruebas evalúa las destrezas de comprensión lectora así como el manejo de vocabulario en general y contiene claves de respuestas para ser seleccionadas a través de dispositivos móviles como tablets, teléfonos celulares o computadores personales. Estas pruebas van acompañadas de tres tests de autoevaluación para averiguar el nivel de estrés, la primera antes de la prueba, la segunda durante la prueba y la tercera después de la prueba. Esta investigación se realizó en el Centro de Idiomas de la Universidad Técnica de Ambato, en donde se aplicó a una muestra de 215 estudiantes, quienes cursaban niveles de inglés, italiano, francés y chino mandarín. Para la recolección de datos se utilizaron las técnicas de la encuesta aplicadas en test, en la que la información que se generó fue tabulada y representada mediante gráficos y tablas estadísticas; estos resultados nos dieron a conocer una correlación negativa significativa entre el nivel de estrés generado y el rendimiento académico, y que existe mayor grado de estrés durante la prueba, bajando el nivel de estrés antes de la prueba y un mínimo nivel de estrés al finalizar la prueba; lo que implica que los estudiantes frente a una evaluación utilizando aplicaciones tecnológicas, existe poca tolerancia de comportamientos frente a las evaluaciones que realizan.

Palabras claves: Aplicaciones tecnológicas, estrés académico, desempeño académico, evaluación en línea.

\section{Introducción.}

Como expresan Ponce y Moran vivimos en un mundo globalizado en donde el aprender idiomas es parte de la sabiduría moderna que nos permiten un mundo de posibilidades, como el poder 
interactuar con personas de todo el mundo (Ponce \& Moran, 2017). Según López Neira vivimos en un mundo tecnológico, que, entre otras cosas, se puede acceder a la aplicación de métodos usando la tecnología para evaluar el idioma extranjero y utilizando algunas herramientas informáticas, para que estos conocimientos sean aplicables se pueden utilizar las tabletas, los celulares, las computadoras, y otros instrumentos, que sin lugar a dudas acceden cada vez a un uso frecuente en la educación universitaria (López, 2017). Para aprender un idioma extranjero la tecnología es de mucha utilidad así lo afirman Ponce y Moran, quienes manifiestan que saliendo de cualquier forma tradicional de enseñanza, la tecnología va en relación con las nuevas generaciones de jóvenes, así como avanza la ciencia y los procesos tecnológicos también los estudiantes usan varios dispositivos tecnológicos para el progreso de la práctica del lenguaje y la escritura, por eso, abre las puertas al mundo actual, permitiendo a los estudiantes entablar relaciones con otras personas y demostrar sus capacidades y habilidades personales (Ponce \& Moran, 2017). La tecnología permite varias actividades académicas, lo que significa que facilita el proceso de enseñanza - aprendizaje, según Escalante Patricia dice que el uso tecnológico es más común en pizarras inteligentes, diccionarios electrónicos, interacción social y el mismo acceso al internet con laptops, que permiten hacer evaluación de los aprendizajes de varias maneras. (Escalante, 2015). Según manifiesta Hermosa Paola que la educación está basada en trasmitir el mensaje también a través de la forma digital o también llamado e-learning, en la que todos los actores están involucrados, dejando huella impactante como contribución a la transformación de la enseñanza - aprendizaje y como conciliación con el progreso tecnológico, así el proceso educativo es más halagador para todos sus actores. (Hermosa, 2015). Además, el docente competente es quien propicia los aprendizajes en el aula, para que los estudiantes utilicen los medios electrónicos, y las TICs, según afirman León, Navío y Barroso, también el docente debe estar capacitado para que entregue sus conocimientos al servicio de los estudiantes, y de esta manera ellos, sean portadores de las buenas prácticas educativas (Leon, Navío, \& Barrosso, 2016).

El estrés es un término muy de moda y se ha entendido como "una reacción fisiológica del organismo en el que entran en juego diversos mecanismos de defensa para afrontar una situación que se percibe como amenazante o de demanda incrementada" (https://es.wikipedia.org/wiki/Estr\%C3\%A9s), es un asunto interactivo en la cual la persona soporta o se defiende de una situación, en donde un nivel moderado de estrés entra 
en la normalidad, hasta que la persona se adapte a una situación (Vidal, Muntaner, \& Sampol, 2018). Según Zarate Nikell y Soto María, los generadores de estrés no solo pueden provenir desde dentro de las aulas, sino también desde fuera, desde los mismos hogares o desde cualquier ambiente, y se incrementa cuando en los estudios universitarios existe presión por las pruebas o exámenes, sean estos utilizando papel y lápiz o utilizando la tecnología. (Zárate, Soto, Castro, \& Quintero, 2017).

Zárate y otros, manifiestan también que el estrés es de forma psicológica y depende de las circunstancias que se presentan como respuestas, llamadas o indicios palpables a las circunstancias de la vida cuotidiana (Zárate, y otros, 2018). Los escenarios tecnológicos e interactivos dentro y fuera del aula favorecen el aprendizaje, pero a la vez causan también malestar en los estudiantes, esta molestia llamada estrés aumenta aún más cuando se trata de pruebas o exámenes elaboradas utilizando la aplicación de herramientas tecnológicas. Según Castillo, Chacón y Diaz, la angustia que manifiestan los estudiantes de la carrera de enfermería y tecnología médica en carrera de universitaria de salud provocan una molestia psíquica, y más aún si se refiere a las evaluaciones para tener mejores notas y la capacidad de soportar muchas dificultades y los niveles de ansiedad (Castillo, Chacón, \& Díaz, 2016). Los estudios superiores, las carreras en las facultades y el aprendizaje de idiomas en las universidades generan un nivel de presión a la que los estudiantes se sienten sometidos, es bien cierto que a algunos les afecta más y a otros menos, pero todos sienten la influencia especialmente cuando llegan las evaluaciones, así lo afirman Toribio y Franco cuando manifiestan que al cursar una carrera universitaria los estudiantes se sienten sometidos al estrés, a los problemas cognitivos, emocionales y fisiológicos (Toribio \& Franco, 2016).

Según Matalinares y otros, dentro de las aulas de clase en la universidad se crea una inestabilidad y una ansiedad cuando se realizan tareas escolares, esta es una época en la que los estudiantes tienen una carga pesada y mucha demanda interna y externa y ellos se enfrentan a diversas posibilidades, y por ende, aparece el estrés como una defensa a cualquier situación personal, de estudio, familiar o ambiental que se pueda presentar (Matalinares, y otros, 2016). También Domínguez Sergio manifiesta que varios eventos académicos, especialmente los exámenes producen presión en los estudiantes que van creciendo de forma exponente "la situación pre examen puede ubicarse en la fase anticipatoria, en la que el estudiante se prepara y analiza la forma 
más eficaz de regular sus emociones frente a la evaluación. En esta etapa la incertidumbre tiende a ser alta, facilitando la aparición de emociones negativas" (Dominguez, 2016, pág. 40).Según Pérez, Vánela y Smith el estrés producido en el ámbito educativo en estudiantes de medicina, especialmente por la carga de los exámenes y por los diferentes horarios que están sometidos, son los principales estresores académicamente "Las principales reacciones al estrés académico manifestados por los sujetos del estudio incluyen: -Psicológicas: ansiedad, desesperación y falta de concentración. -Físicos: dolor de cabeza, fatiga crónica y trastornos del sueño. Comportamentales: tendencia a polemizar, falta de interés para realizar tareas. El cambio en la ingesta de alimentos también resultó relevante.” (Pérez, Vanela, \& Arcelia, 2019, pág. 95).

Según los autores Vallejo, Ajo y Plaza muchos estudiantes tienen el desafío ante las tareas, y pruebas, además por conseguir notas más altas, esta situación produce estrés, con una serie de manifestaciones que muchas veces son visibles y otras no lo son, por lo que el estrés se manifiesta como un proceso psicosocial que afecta a la persona con sus manifestaciones físicas, sino también revelaciones de carácter social y personal. (Vallejo, Aja, \& Plaza, 2017, pág. 222). Según Vidal, Muntaner y Palou cualquier situación adversa puede desencadenar un proceso de estrés, y peor aun cuando la persona no puede enfrentar a esa situación, que produce efectos fisiológicos y se puede decir que el estrés es parte de la existencia de los estudiantes universitarios y afecta al rendimiento y sus notas académicamente hablando. (Vidal, Muntaner, \& Palou Sampol, 2018).

\section{Metodología}

El presente trabajo investigativo se lo realizó a partir del proyecto "La aplicación de métodos estandarizados, no estandarizados y tecnológicos de evaluación de idioma extranjero en relación al nivel de estrés que estos generan en los estudiantes universitarios". De la cual se abre una línea de investigación realizando un análisis comparativo de una encuesta que contiene varias preguntas aplicadas antes del examen, durante el examen y después del examen usando las aplicaciones tecnológicas mencionadas, con la participación de estudiantes de inglés, italiano, francés y chino mandarín. Estos resultados se tabularon y se analizaron con el propósito de determinar el nivel de estrés que dichos exámenes causan a los estudiantes del Centro de Idiomas de la Universidad Técnica de Ambato. 


\section{Participantes}

Los estudiantes participantes fueron 215, pertenecientes a los idiomas: Inglés, Italiano, Francés y Chino Mandarín, y que fueron seleccionados de 45 paralelos del Centro de idiomas de la Universidad Técnica de Ambato, cuyo universo total fue de 1350 estudiantes, cuyas edades están comprendidas entre 18 y 30 años. Los estudiantes corresponden a los niveles A1, A2, B1 y B1+. Los datos se tomaron en el ciclo académico septiembre 2018 y febrero 2019.

\section{Instrumentos}

Para la recopilación de datos y determinación del nivel de estrés que los estudiantes presentan se utilizó un cuestionario previamente validado del Inventario SISCO del Stress Académico (Barraza Macias, 2007), a través la aplicación Google forms y completada por la muestra de estudio en tres días consecutivos, en la que contenían para cada día tres cuestionarios diferentes, un cuestionario antes del examen llamado de los aprendizajes, otro cuestionario durante el examen llamado como los aprendizajes y un último cuestionario llamado para los aprendizajes. Simultáneamente se utilizó un cuestionario con contenidos de la asignatura estudiados en clase, para realizar la evaluación de aprendizajes. Este cuestionario también fue realizado utilizando la aplicación StarQuiz Netclient.

\section{Procedimiento}

Se recogió la información mediante links, tanto de las pruebas de conocimientos como de la encuesta aplicada para medir el nivel de estrés, de la siguiente manera: Primero se aplicó el cuestionario de medición de estrés, antes de una evaluación de conocimientos; luego se inició con el desarrollo de la prueba de conocimientos. Cuando los encuestados habían contestado la mitad de la Prueba, se aplicó nuevamente el cuestionario SISCO, para determinar variaciones en el nivel de estrés a medida como se desarrollaba la prueba. Luego se pidió a los estudiantes que continúen desarrollando la prueba de conocimientos, y finalmente, al termino de dicha prueba, se aplicó nuevamente el cuestionario SISCO de medición del nivel de estrés, con el fin de observar y comparar los resultados. Dando respuesta a cada una de las preguntas planteadas en el cuestionario SISCO, en una escala de 0 al 5 en las primeras tres preguntas Antes de rendir la evalución: 1. ¿Cuál es su nivel de preocupación o nerviosismo? 2. Señale el nivel de nerviosismo que le causa las siguientes situaciones 3. ¿Qué tipo de reacciones físicas, psicológicas y comportamentales 
evidencia en la evaluación? 4. ¿Cuál de las siguientes estrategias y con qué frecuencia las usa Usted para enfrentar la situación que le causa preocupación o nerviosismo antes de rendir la evaluación? Durante la Evaluación y en una escala de 0 al 5 en las primeras tres preguntas 1 . En este momento ¿Cuál es su nivel de preocupación y nerviosismo? 2. Señale el nivel de nerviosismo que le está causando cada una de las siguientes situaciones 3. Señale el tipo de reacciones físicas, psicológicas y comportamentales que se evidencia al momento de la evaluación? 4. ¿Cuál de las siguientes estrategias está usando Usted para enfrentar la situación que le causan preocupación o nerviosismo en este momento? Después de la Evaluación: y en una escala de 0 al 5 en las primeras tres preguntas 1. Luego de la presente evaluación, ¿Cuál es su nivel de preocupación o nerviosismo? 2. Señale el nivel de nerviosismo que le causó cada una de las siguientes situaciones? 3. Señale el tipo de reacciones físicas, psicológicas y comportamentales que se evidencia luego de la evaluación. 4. ¿Cuál de las siguientes estrategias utilizo para disminuir su preocupación o nerviosismo luego de la evaluación?

\section{Análisis Estadístico}

Los análisis estadísticos fueron analizados mediante el paquete estadístico SPSS-21 para Windows, y se realizaron los análisis descriptivos y representados en tablas, en la cual se muestra los porcentajes de las variables planteadas.

\section{Resultados}

Los resultados obtenidos a partir de los datos del cuestionario utilizado muestran que

\section{Evaluación de aprendizajes}

Para el análisis de confiabilidad de la encuesta, se corrió el estadístico Alfa de Cronbach, la cual se tuvo un valor de 0,926, esto nos indica que tenemos una excelente confiabilidad de la encuesta realizada.

Con el objetivo de medir el nivel de preocupación o nerviosismo antes, durante y después de la evaluación se corrió la prueba no paramétrica de Friedman, la cual tenemos un p-valor de 0,296 lo cual nos quiere decir que no hay diferencias significativas en los tres momentos, y se mantienen con una mediana de 2 en una escala del 0 al 5 . 
Con el objetivo de ver las preguntas que mayor nerviosismo provoca en los estudiantes en el momento de realizar una evaluación se corrió un estadístico descriptivo de las medianas y medias, y se presenta las siguientes preguntas, que se detallan en la siguiente tabla:

Tabla No. 1. Estrategias para enfrentar

\begin{tabular}{lll}
\hline SITUACIONES & Momento & Mediana \\
\hline $\begin{array}{l}\text { La personalidad y el carácter del profesor. } \\
\begin{array}{l}\text { Desconocer el tipo de evaluación aplicada } \\
\text { por el docente. }\end{array}\end{array}$ & $\begin{array}{l}\text { Después } \\
\text { Estrategias para enfrentar estress }\end{array}$ & 3 \\
$\begin{array}{l}\text { Habilidad asertiva (Aceptación de la } \\
\text { realidad) }\end{array}$ & Antes- después & 3 \\
$\begin{array}{l}\text { Elaboración de un plan mental para recordar } \\
\text { los contenidos más importantes. }\end{array}$ & Antes & 3 \\
\hline
\end{tabular}

Elaborado por: Grupo Investigador

Como se puede apreciar, el nivel de estrés es bastante alto, debido a la personalidad del docente, así como también al tipo de evaluación aplicada por el profesor. Entre las estrategias mas utilizadas para enfrentar el estrés, los estudiantes desarrollan planes personales para aceptar la realidad y ayudarse mutuamente.

\section{Evaluación para aprendizajes}

Para el análisis de confiabilidad de la encuesta, se corrió el estadístico Alfa de Cronbach, la cual se tuvo un valor de 0,896 , la cual nos indica que tenemos una excelente confiabilidad de la encuesta realizada.

Con el objetivo de medir el nivel de preocupación o nerviosismo antes, durante y después de la evaluación se corrió la prueba no paramétrica de Friedman, la cual tenemos un p-valor de 0,002 lo cual indica que existen diferencias significativas en los tres momentos, se calcula las medias y medianas para ver en qué momento hay mayor nerviosismo en la escala del 0 al 5. 
Tabla No. 2. Estadísticos

\begin{tabular}{|c|c|c|c|}
\hline \multicolumn{4}{|c|}{ Estadísticos } \\
\hline & Antes & Durante & Después \\
\hline $\mathrm{N}$ & 215 & 215 & 215 \\
\hline Media & 2,70 & 2,51 & 2,09 \\
\hline Mediana & 3,00 & 3,00 & 2,00 \\
\hline
\end{tabular}

Elaborado por: Grupo Investigador

Se aprecia claramente que el nivel de nerviosismo es alto antes de rendir la evaluación, durante la evaluación sigue con un nivel más bajo de nerviosismo, y después de la evaluación, ya es leve.

Con el objetivo de ver las preguntas que mayor nerviosismo provocan en los estudiantes en el momento de realizar una evaluación se corrió un estadístico descriptivo de las medianas y medias, y se presenta las siguientes preguntas, que se detallan en la siguiente tabla:

Tabla No. 3. Situación

\begin{tabular}{|c|c|c|}
\hline SITUACIONES & Momento & Mediana \\
\hline $\begin{array}{l}\text { Expectativa sobre contenido de la } \\
\text { evaluación. } \\
\text { Estrategias para enfrentar estress }\end{array}$ & Antes- después & 3 \\
\hline $\begin{array}{l}\text { Habilidad asertiva (Aceptación de la } \\
\text { realidad) }\end{array}$ & Antes- después & 3 \\
\hline $\begin{array}{l}\text { Elaboración de un plan mental para recordar } \\
\text { los contenidos más importantes. } \\
\text { Tabla 3: Grupo investigador }\end{array}$ & Antes - después & 3 \\
\hline
\end{tabular}

Elaborado por: Grupo Investigador

\section{Evaluación como medio de aprendizajes}

Para el análisis de confiabilidad de la encuesta, se corrió el estadístico Alfa de Cronbach, la cual se tuvo un valor de 0,908 , la cual nos indica que tenemos una excelente confiabilidad de la encuesta realizada.

Con el objetivo de medir el nivel de preocupación o nerviosismo antes, durante y después de la evaluación se corrió la prueba no paramétrica de Friedman, la cual tenemos un p-valor de 0,006 lo 
cual nos quiere decir que existe diferencias significativas en los tres momentos, se calcula las medias y medianas para ver qué momento hay mayor nerviosismo en la escala del 0 al 5.

Tabla No. 4.Grupo investigador

\begin{tabular}{llll}
\hline Estadísticos & & & \\
\hline & Antes & Durante & Después \\
$\mathrm{N}$ & 215 & 215 & 215 \\
Media & 2,97 & 2,55 & 2,65 \\
Mediana & 3,00 & 3,00 & 3,00
\end{tabular}

Elaborado por: Grupo Investigador

Se aprecia claramente que el nivel de nerviosismo es antes de rendir la evaluación con una media de 2,97, durante la evaluación sigue con un nivel más bajo de nerviosismo, y después de la evaluación aumenta el nivel de nerviosismo 2,65.

Con el objetivo de ver las preguntas que mayor nerviosismo provoca en los estudiantes en el momento de realizar una evaluación se corrió un estadístico descriptivo de las medianas y medias, y se presenta las siguientes preguntas, que se detallan en la siguiente tabla:

Tabla No. 5. Situación

\begin{tabular}{|c|c|c|}
\hline SITUACIONES & Momento & Mediana \\
\hline $\begin{array}{l}\text { Desconocer el tipo de evaluación } \\
\text { aplicada por el docente. } \\
\text { Expectativa sobre contenido de la } \\
\text { evaluación. } \\
\text { Estrategias Para Enfrentar Estress }\end{array}$ & $\begin{array}{l}\text { Antes - después } \\
\text { Antes- durante - después }\end{array}$ & 3 \\
\hline $\begin{array}{l}\text { Habilidad asertiva (Aceptación de la } \\
\text { realidad) }\end{array}$ & Antes- después & 3 \\
\hline $\begin{array}{l}\text { Elaboración de un plan mental para } \\
\text { recordar los contenidos más importantes. }\end{array}$ & Antes - después & 3 \\
\hline Elogios a sí mismo & Después & 3 \\
\hline
\end{tabular}

Elaborado por: Grupo Investigador 
Según (Barraza, 2008) este conjunto de indicadores se articulan en las personas, causando un desequilibrio sistémico, el cual se manifiesta de diferentes formas en cantidad y en variedad en cada persona. Ante este desequilibrio, el individuo pone en marcha diversas estrategias para afrontar y restaurar dicho equilibrio. (Barraza) sugiere las siguientes: habilidad asertiva; elogios a sí mismo; distracciones evasivas como por ejemplo la religiosidad, otros llegan a solicitar ayudad profesional, o simplemente lo toman con un buen sentido del humor, y elaboran un plan para enfrentarlo, como ya se ha comprobado en la presente investigación.

Los resultados de esta experiencia fueron muy satisfactorios en relación con la evaluación de aprendizajes en una clase de idioma extranjero. Como se aprecian en las tablas de reporte de resultados, cada uno de los evaluados tienen diferentes estrategias para enfrentar el estrés causado por las evaluaciones de conocimientos.

Tabla No. 6. Evaluación

\section{ANTES DE LA EVALUACIÓN}

1. Antes de la evaluación. Cuál es el nivel de preocupación o nerviosismo?

\begin{tabular}{|l|l|l|l|l|l|}
\hline 0 & 1 & 2 & 3 & 4 & 5 \\
\hline & & & & & \\
\hline
\end{tabular}

2. En una escala del (0) al es nada , y (5) es mucho, señale el nivel de

\begin{tabular}{|l|l|l|l|l|l|}
\hline 0 & 1 & 2 & 3 & 4 & 5 \\
\hline
\end{tabular}

SITUACIONES

\begin{tabular}{|l|l|l|l|l|l|l|}
\hline $\begin{array}{l}\text { Asignación del compañero de } \\
\text { trabajo }\end{array}$ & & & & & & \\
\hline $\begin{array}{l}\text { La personalidad y el carácter del } \\
\text { profesor }\end{array}$ & & & & & & \\
\hline $\begin{array}{l}\text { Desconocer el tipo de valuación } \\
\text { aplicada por el docente }\end{array}$ & & & & & & \\
\hline $\begin{array}{l}\text { Expectativa sobre el contenido de la } \\
\text { evaluación }\end{array}$ & & & & & & \\
\hline Otra (especifique) & & & & & & \\
\hline
\end{tabular}

3. En una escala del (0) al es nada, y (5) es mucho, señale el tipo de reacciones

\begin{tabular}{|l|l|l|l|l|l|}
\hline 0 & 1 & 2 & 3 & 4 & 5 \\
\hline
\end{tabular}

REACCIONES FISICAS

Dolores de cabeza o migraña

Problemas de digestión. Dolor abdominal o diarrea

\begin{tabular}{|l|l|l|l|l|l|}
\hline & & & & & \\
\hline & & & & & \\
\hline
\end{tabular}




\begin{tabular}{|l|l|l|l|l|l|l|}
\hline $\begin{array}{l}\text { Rascarse, morderse las uñas, } \\
\text { frotarse, etc. }\end{array}$ & & & & & & \\
\hline Algún tipo de temblor corporal & & & & & & \\
\hline Otras (especifique) & & & & & & \\
\hline
\end{tabular}

\section{REACCIONES}

\section{PSICOLOGICAS}

\begin{tabular}{|l|l|l|l|l|l|l|}
\hline $\begin{array}{l}\text { Inquietud (incapacidad de relajarse y } \\
\text { estar tranquilo) }\end{array}$ & & & & & & \\
\hline $\begin{array}{l}\text { Sentimientos de depresión y tristeza } \\
\text { (decaído) }\end{array}$ & & & & & & \\
\hline $\begin{array}{l}\text { Ansiedad, angustia o } \\
\text { desesperación }\end{array}$ & & & & & & \\
\hline problemas de concentración & & & & & & \\
\hline $\begin{array}{l}\text { Sentimiento de agresividad y aumento } \\
\text { de irritabilidad }\end{array}$ & & & & & & \\
\hline Otras (especifique) & & & & & & \\
\hline
\end{tabular}

\section{REACCIONES}

\section{COMPORTAMENTALES}

Conflictos o tendencia a polemizar o discutir

Aislamiento de los demás desgano para realizar la evaluación

Otras( especifique)

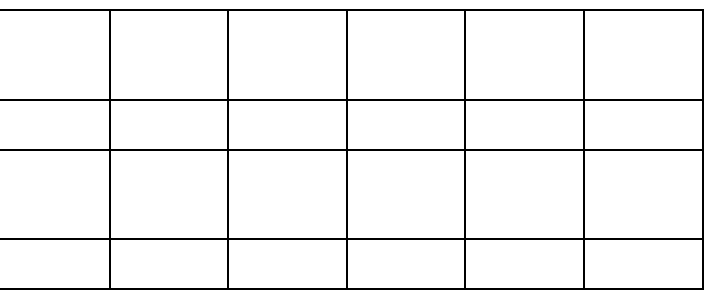

4. En una escala del (1) al (5) donde (1) es nunca, (2) rara vez, (3) es algunas veces

\begin{tabular}{|l|l|l|l|l|l|}
\hline 0 & 1 & 2 & 3 & 4 & 5 \\
\hline
\end{tabular}

\section{ESTRATEGIAS PARA}

\section{ENFRENTAR EL ESTRÉS}

\begin{tabular}{|l|l|l|l|l|l|l|}
\hline habilidad asertiva & & & & & & \\
\hline $\begin{array}{l}\text { Elaboración de un plan mental para } \\
\text { recordar los contenidos }\end{array}$ & & & & & & \\
\hline Elogios a si mismo & & & & & & \\
\hline la religiosidad (oraciones a Dios) & & & & & & \\
\hline Otras (especifique) & & & & & & \\
\hline
\end{tabular}

\section{Recomendaciones}

Para una mejor aplicación de métodos tecnológicos de evaluación de aprendizajes en una clase de idioma extranjero, es necesario que: 
- Los estudiantes estén familiarizados con dichas aplicaciones y/o plataformas de evaluación. Para ello también es necesario que el salón de clase cuente con el servicio de internet inalámbrico.

- Los docentes de Idioma extranjero deben considerar la implementación de estrategias de evaluación en su planificación, dando uso a los dispositivos electrónicos que los estudiantes disponen (celular y/o tablets).

- También se debe implementar un plan de capacitación a los docentes de idioma extranjero, en el uso y manejo de ciertas aplicaciones telefónicas que le permitirán evaluar de mejor forma los aprendizajes de sus estudiantes.

- Se debe considerar además que el uso de la tecnología computacional es un elemento integral para el desarrollo de un segundo idioma.

- Los docentes de idioma extranjero deben además promover en sus estudiantes, el uso de aplicaciones y plataformas para facilitar el aprendizaje del mismo fuera del salón de clase, es decir cuando el estudiante se encuentre en su hogar.

- Las universidades deben invertir en la implementación de tecnología y facilitar el uso de estos recursos para todos los usuarios dentro del campus.

- Los instructores de idioma extranjero, deben convertirse en amigos y compañeros en el uso de herramientas a aplicaciones tecnológicas (Pourhossein Gilakjani, \& Sabouri, 2017)

\section{Conclusiones}

Se concluye que la información generada demuestra que existe un mayor nivel de preocupación, nerviosismo y estrés antes de la evaluación de aprendizajes, y que para la presente investigación se utilizaron recursos y plataformas tecnológicas tales como StarQuiz Netclient, Google Forms, Socrative, así mismo se observa una disminución de la intensidad de estrés durante la evaluación y finalmente se puede notar al estudiante mucho más relajado al final de la evaluación. Esto evidencia que los estudiantes del Centro de idiomas frente a la aplicación de evaluaciones a través de recursos tecnológicos, se muestran predispuestos al reto de ser evaluados, los cuales no generan niveles de estrés y nerviosismo.

- En el Centro de Idiomas se debe fomentar la creación de talleres formativos para que los estudiantes a través de metodologías y estrategias puedan enfrentar situaciones difíciles 
que provoquen estrés. Estos espacios de dialogo también deben incluir a docentes que permitan crear en sus aulas estados de mucha tranquilidad ante un examen utilizando la tecnología.

- Ante el nivel de preocupación que se denota en los resultados, se deben seguir investigando para determinar si hay modificaciones en todos los niveles del estudio de idioma extranjero, comparándolos entre todos, para así, relacionar diferentes variables demostradas en todas las etapas de la vida estudiantil.

- Reflexionando sobre los datos obtenidos, el estrés que se genera en las evaluaciones disminuyen ostensiblemente a medida que el examen y/o evaluación de aprendizajes va finalizando; sin embargo, cada estudiante debe construir nuevas formas de responsabilidad, entendiéndose que al controlar su estrés, está alcanzando un peldaño más hacia el logro de una madurez profesional, por lo tanto el estudiante, debe demostrar una superación, crecimiento emocional y desarrollo integral en su etapa estudiantil.

\section{Bibliografía}

Barraza. (2008). El estrés académico en alumnos de maestría y sus variables moduladoras: un diseño de diferencia de grupos [Versión electrónica]. Avances en Psicología Latinoamericana, 2 (26), 270-289.

Barraza Macias, A. (2007). Propiedades Psicometricas del Inventario SISCO del Estres Academico. Biblioteca Virtual de la Psicologia Cientifica.com.

Castillo, C., Chacón, T., \& Díaz, G. (2016). Ansiedad y fuentes de estrés académico en estudiantes de carreras de la salud. Investigación en educación médica, 230 -237.

Dominguez, S. (2016). Afrontamiento ante la ansiedad pre-examen y autoeficacia académica en estudiantes de ciencias de la salud. Educación Médica, 39 - 42.

Escalante, P. (2015). Estado actual de la integración de tecnologías digitales en la enseñanza de idiomas enla Escuela de Lenguas Modernas dela Universidad de Costa Rica. Revista de Lenguas Modernas, 329-348.

Fuentes, J., Esteban, F., \& González, M. (2016). Sobre las dimensiones identitarias esenciales del profesorado universitario en el contextopedagógico-tecnológico contemporáneo. Education in the Knowledge Society, 39-53.

Hermosa, P. (2015). Influencia de las tecnologías de información y comunicación (TIC) en el proceso enseñanza-aprendizaje: una mejora de las competencias digitales. General José María Córdov, 121-132. 
Leon, R., Navío, A., \& Barrosso, J. (2016). LAS COMPETENCIAS DEL PROFESORADO UNIVERSITARIO DESDE EL MODELOTPACK (CONOCIMIENTO TECNOLÓGICO Y PEDAGÓGICO DEL CONTENIDO). Revista de Medios y Educación, 105-119.

López, N. (2017). Indagación en la relación aprendizaje-tecnologías digitales. Tecnologías de la informacióny la comunicación en la Pedagogía, 91 - 105.

Matalinares, M., Diaz, G., Raymundo, O., Baca, D., Uceda, J., \& Yaringaño, J. (2016). Afrontamiento del estrés y bienestar psicológico en estudiantes universitarios. PERSONA, 105 - 126.

Pérez, M., Vanela, Y., \& Arcelia, S. (2019). Caracterización del estrés académico percibido por estudiantes de las ciencias de la salud y los mecanismos que utilizan para afrontarlo. Veraguas, 2018. Visión Antataura, 79-97.

Ponce, L., \& Moran, J. (2017). LAS TIC COMO MDEIO DE APRENDIZAJE DE LOS IDIOMAS. Cognosis, 23 - 30.

Ponce, L., \& Moran, J. (2017). LAS TIC COMO MEDIO DE DE APRENDIZAJE DE LOS IDIOMAS. Cognosis, 23 - 30.

Pourhossein Gilakjani, \& Sabouri. (2017). A review of the literature on the integration of technology into the learning and teaching of English language skills. International Journal of English Linguistics, , 95-106.

Toribio, C., \& Franco, S. (2016). Estrés Académico: El Enemigo Silencioso del Estudiante. Salud y Administración, 11 - 18.

Vallejo, M., Aja, J., \& Plaza, J. (2017). Estrés percibido en estudiantes universitarios: influencia del burnout y del engagemet académico. REVISTA INTERNAL DE INVESTIGACIÓN E INNOVACIÓN EDUCATIVA, 220 - 236.

Vidal, J. (2018). DIFERENCIAS. CONTEXTOS EDUCATIVOS, 223-224.

Vidal, J. (2018). DIFERENCIAS. ECUCACION, 12-34.

Vidal, J., Muntaner, A., \& Palou Sampol, P. (2018). DIFERENCIAS DE ESTRÉS Y AFRONTAMIENTO DEL MISMO SEGÚN EL GENERO Y COMO AFECTA AL RENDIMIENTO ACADÉMICO EN ESTUDIANTES UNIVERSITARIOS. CONTEXTO EDUCATIVOS, 181 -195.

Vidal, J., Muntaner, A., \& Sampol, P. (2018). DIFERENCIAS. CONTEXTOS EDUCATIVOS, 181-195.

Vidal, J., Muntaner, A., \& Sampol, P. (2018). DIFERENCIAS DE ESTRES Y AFRONTAMIENTO DEL MISMO SEGÚN EL GÉNERO Y CÓMO AFECTA AL 
RENDIMIENTO ACADÉMICO EN ESTUDIANTES UNIVERSITARIOS. EDUCACION, 181-195.

Zárate, M., Soto-Decuir, E., Martinez, M., Castro, R., García-Jau, N., \& López, L. (2018). Hábitos de estudio y estrés en estudiantes del área de la salud. Revista de la Fundación Educación Médica, 153 - 157.

Zárate, N., Soto, M., Castro, M., \& Quintero, J. (2017). ESTRÉS ACADÉMICO EN ESTUDIANTES UNIVERSITARIOS: MEDIDAS PREVENTIVAS . Revista de la Alta Tecnología y la Sociedad, 92 - 98. 


\section{Para citar el artículo indexado.}

Llerena Medina, E. G., Rodríguez Hurtado, C. P., \& Valencia Núñez, E. R. (2020). La aplicación de métodos tecnológicos de evaluación de idioma extranjero en relación con el nivel de estrés que estos generan en los estudiantes universitarios. Explorador Digital, 4(2), 5-21. https://doi.org/10.33262/exploradordigital.v4i2.1197

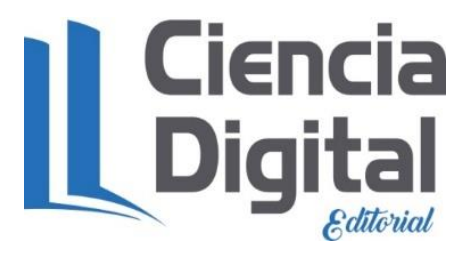

El artículo que se publica es de exclusiva responsabilidad de los autores y no necesariamente reflejan el pensamiento de la Revista Explorador Digital.

El articulo queda en propiedad de la revista y, por tanto, su publicación parcial y/o total en otro medio tiene que ser autorizado por el director o editor de la Revista Explorador Digital.
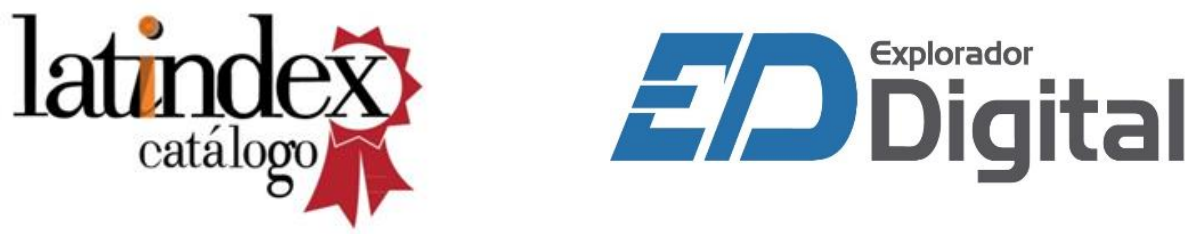